\title{
Increasing Spatial Detail of Burned Scar Maps Using IRS-AWiFS Data for Mediterranean Europe
}

\author{
Fernando Sedano ${ }^{1, *}$, Pieter Kempeneers ${ }^{2}$, Peter Strobl ${ }^{1}$, Daniel McInerney ${ }^{1}$ \\ and Jesús San Miguel ${ }^{1}$
}

1 Land Management and Natural Hazards Unit, Institute for Sustainability and Environment, Joint Research Centre of the European Commission, Via Enrico Fermi, 2749, 21027 Ispra (VA), Italy; E-Mails: peter.strobl@jrc.ec.europa.eu (P.S.); daniel.mc-inerney@jrc.ec.europa.eu (D.M.); jesus.san-miguel@jrc.ec.europa.eu (J.S.M.)

2 Flemish Institute for Technological Research (VITO), Boeretang 200, B-2400 Mol, Belgium; E-Mail: pieter.kempeneers@vito.be

* Author to whom correspondence should be addressed; E-Mail: fersed@gmail.com.

Received: 7 February 2012; in revised form: 6 March 2012 / Accepted: 7 March 2012 /

Published: 15 March 2012

\begin{abstract}
A two stage burned scar detection approach is applied to produce a burned scar map for Mediterranean Europe using IRS-AWiFS imagery acquired at the end of the 2009 fire season. The first stage identified burned scar seeds based on a learning algorithm (Artificial Neural Network) coupled with a bootstrap aggregation process. The second stage implemented a region growing process to extend the area of the burned scars. Several ancillary datasets were used for the accuracy assessment and a final visual check was performed to refine the burned scar product. Training data for the learning algorithm were obtained from MODIS-based polygons, which were generated by the Rapid Damage Assessment module of the European Forest Fire Information System. The map produced from this research is the first attempt to increase the spatial detail of current burned scar maps for the Mediterranean region. The map has been analyzed and compared to existing burned area polygons from the European Forest Fire Information System. The comparison showed that the IRS-AWiFS-based burned scar map improved the delineation of burn scars; in addition the process identified a number of small burned scars that were not detected on lower resolution sensor data. Nonetheless, the results do not clearly support the improved capability for the detection of smaller burned scars. A number of reasons can be provided for the under-detection of burned scars, these include: the lack of a full coverage and cloud free imagery, the time lag between forest fires and image acquisition date and the occurrence of fires after the image acquisition dates. On the other hand, the limited
\end{abstract}


spectral information combined with the presence of undetected cloud shadows and shaded slopes are reasons for the over-estimation of small burned scars.

Keywords: burned scar mapping; remote sensing; medium resolution

\section{Introduction}

Wildfires are a major source of disturbance in natural ecosystems. At a global scale, they impact hydrologic, biogeochemical and atmospheric processes, while at regional and local scales they are a major cause of land use and land cover change. They also affect the productivity and carrying capacity of ecosystems, inflict considerable socio-economic damages and can have deleterious effects on human health [1].

Due to its synoptic view of the landscape and high revisit time, Earth observation data have been widely used for burned scar monitoring. A wide range of information extraction techniques and classification algorithms have been proposed and this has been comprehensively reviewed by Bastarrika [2]. The most notable examples include: spectral thresholding [3] unsupervised classifiers [4,5], linear supervised classifiers [6], non-linear supervised classifiers [7,8], linear unmixing [9] and object-classification [10]. These methodologies have been commonly applied to limited spatial extents or else specific fire events. In contrast, fewer of these methodologies have been performed at regional or continental scales. Working over large extents means that a variety of environmental conditions and landscapes have to be dealt with as well as an increase in the number of satellite images of various qualities and acquisition dates. In this context, Fraser et al. [11] mapped burned areas over Canada using multi-temporal data from the Advanced Very High Resolution Radiometer (AVHRR) combined with thermal hotspots and Zhang et al. [12] produced a monthly burned area map for the Russian Federation from the Satellite Pour l'Observation de la Terre-VEGETATION (SPOT-VGT) satellite sensor.

A number of initiatives have applied coarse resolution remote sensing to monitor burned areas over time at a global scale. The Global VGT burned area product 2000-2007 (L3JRC) [13] and its precursor, Global Burned Area 2000 [14], used SPOT-Vegetation data for the period 2000-2007. The GLOBSCAR [15] was based on European Remote Sensing satellite 2 Along-Track Scanning Radiometer 2 (ERS2-ATSR2) for the year 2000, while the GlobCarbon project [16] for the period 1998-2007 was based on ERS2-ATSR2 and ENVISAT-Advanced Along-Track Scanning Radiometer (AATSR) imagery. Currently, the Moderate Resolution Imaging Spectroradiometer (MODIS) Burned Area Product is the only sensor that operationally provides global monthly information at a spatial resolution of 500-m [11,17].

With the technological developments of the last decade, there has been a proliferation of high and medium resolution Earth observation sensors [18]. The integration of data from several sensors of similar characteristics is providing new opportunities to map burned scars over large extents. Occasional higher resolution products could complement information from medium resolution sensors, combining the temporal and spatial dimensions offered by satellite remote sensing. 
The Mediterranean region in Europe is highly susceptible to wildfires. Approximately 60,000 fires occur every year in this region, burning on average, about half a million hectares of forest and natural vegetation [19]. The Rapid Damage Assessment module of the European Forest Fire Information System (EFFIS-RDA) managed by the European Commission's Joint Research Centre (EC-JRC) provides daily estimates of the areas affected by forest fires during the fire season. EFFIS-RDA allows burned scars of approximately 50 ha or larger to be consistently mapped across Europe, while smaller burned scars may be registered under specific circumstances. Burned scars larger than 50 ha account for about $75 \%$ of the total area burned every year in the Mediterranean region [20]. In regions such as the north west of the Iberian Peninsula and Italy, burned scars, smaller than 50 ha, represent a relevant proportion of the total burned area. Therefore, monitoring these fires is essential from an ecological, social and economic perspective. Improving burned scar mapping capabilities to consistently map areas larger than 10 ha (covering 90\% of the fires over Europe) would provide valuable information on the impact of forest fires [21]. Furthermore, the European Parliament, in its resolution of 2006, called for an improvement in the assessment of burned areas already provided by EFFIS with the use of higher spatial resolution datasets. Such an improvement would lead to a more accurate quantification of the spatial extent and distribution of damages, thereby allowing for more efficient post-fire management.

The research presented in this paper evaluates the feasibility of burned scar mapping over large regions using medium spatial resolution remote sensing data from the Advanced Wide Field Sensor (IRS-AWiFS) sensor. It is carried out across the Mediterranean region using data acquired between August and September 2009. Although IRS-AWiFS images have already been applied for burned scar detection at sub-national level [22], they have not been used for such purposes at regional or sub-continental scales. This study aims to identify some of the main advantages and disadvantages of medium spatial resolution burned scar mapping over existing coarse resolution products.

\section{Study Area}

The area of interest is Mediterranean Europe (Med-EU) (Figure 1), which covers 2.4 million km² and includes Portugal, Spain, southern France, Italy, Greece, and Turkey, those being the most fire prone countries in Europe. According to the United Nations Food and Agriculture Organization (UN FAO) Global Ecological Zones Map [23], the region contains five out of seven main ecological zones in Western Europe: temperate oceanic forest, subtropical dry forest, temperate mountain systems, temperate continental forests and subtropical mountain systems. The summer season starts in June and ends in September. However, depending on the specific geographic location and weather conditions of a given year, the fire season can potentially start in April or May and last until October or November. The frequency of forest fires in this region has its peak in July and August, corresponding to the highest overall annual temperatures and periods of lowest precipitation.

In 2009, the number of recorded fires in Portugal, Spain, southern France, Italy and Greece was 52,795 fires, which affected a total area of 323,896 ha. These figures are below average for the last 29 years [24]. 
Figure 1. (A) Location map (ETRS89/LAEA projection) and coverage of the 61 IRS-AWiFS scene dataset. (B) Number of valid observations after applying cloud and cloud shadow, slope and water masks. The color scale represents the number of valid observations. Red tones indicate a large number of valid observations while blue tones indicate a low number of valid observations.

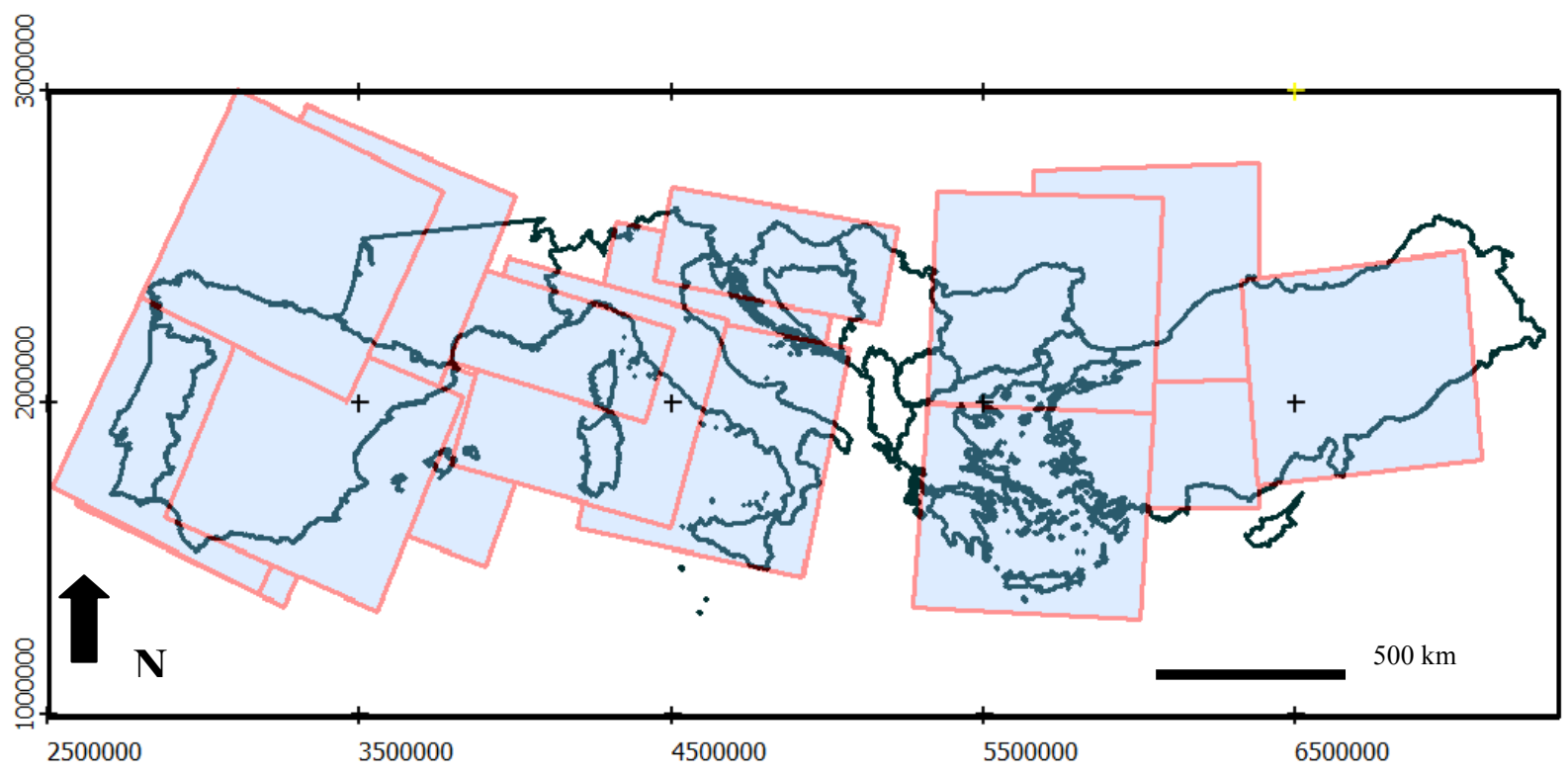

(A)

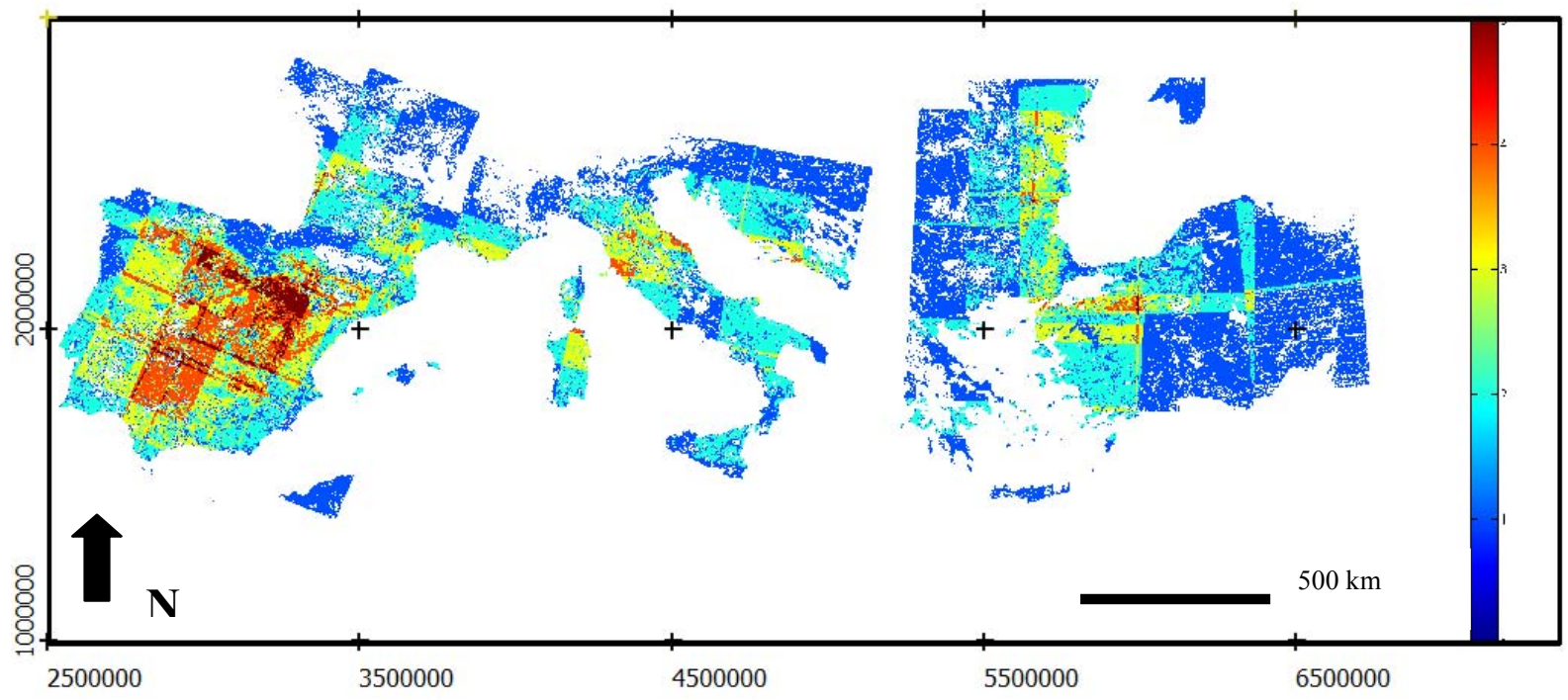

(B)

\section{Data}

\subsection{Satellite Imagery}

Images from the IRS-AWiFS were used in this study. The sensor operates in four spectral bands (green, red, near infrared and short wave infrared) and acquires imagery with a spatial resolution of $56 \mathrm{~m}$ at nadir with a swath of $740 \mathrm{~km}$ [25]. The original AWiFS images were resampled to a $50 \mathrm{~m}$ LAEA/ERTS89 grid, following the European Commission INSPIRE Directive [26], as the main aim of 
this work is the provision of official statistics of burned areas for the use of European Commission services and national forest fire administrations.

In order to capture the maximum possible number of burned scars that occurred during the 2009 fire season the acquisition window spanned from mid August to late October. The final dataset contained 61 AWiFS scenes with some degree of overlap. The overlap of the scenes varies within the study area. In general western Mediterranean Europe has more overlap than Eastern Med-EU (Figure 1). Despite of the length of the acquisition frame, the final dataset did not provide a complete coverage of Mediterranean Europe since cloud-free imagery was not available for part of the Balkans.

\subsection{Burned Area Training Data}

The first stage for the estimation of burned scars from IRS-AWiFS data was performed with a supervised classification algorithm. The original training data for the supervised classification were obtained from MODIS-based polygons generated by the Rapid Damage Assessment (RDA) in EFFIS. This product provides spatially consistent information about burned areas in Europe. RDA burned areas are derived from the $250 \mathrm{~m}$ spatial resolution MODIS data. Burned area perimeters were extracted using an automated supervised classification of the data, which were then refined through visual interpretation of the images. Active fires detected by MODIS, forest fire news, as well as land cover datasets were used to refine the original classification and to eliminate possible mistakes made during the automatic classification (http://effis.jrc.ec.europa.eu). The system records burned areas for fires of about 50 ha or larger, although a large number of fires smaller than this threshold are also mapped. The forest fire perimeters for year 2009 were converted to $50 \mathrm{~m}$ raster files in the LAEA/ERTS89 projection prior to their combination with the AWiFS scenes.

\subsection{Ancillary Data}

The production of the burned scar map from AWiFS imagery required several masks (see Section 4). Each mask was created using a number of ancillary spatial datasets that include:

- A 100 m spatial resolution digital elevation model (DEM) resampled from the Version 3 of the Shuttle Radar Topographic Mission (SRTM) 90 m Digital Elevation Data [27]. For Eurasia the absolute height error and absolute geolocation error of the SRTM DEM have been estimated in $6.2 \mathrm{~m}$ and $8.8 \mathrm{~m}$ respectively [28]. Until a full validation of the ASTER Global Digital Elevation Model is carried out, the SRTM-DEM is the most complete and accepted high-resolution digital topographic database of Earth.

- The Forest Type Map 2006 (FTYP2006) at $25 \mathrm{~m}$ spatial resolution developed by the EC-JRC [28]. This product provides information about the distribution of broadleaved and coniferous forests. In addition to this information, this product also includes a water class at a spatial resolution of $25 \mathrm{~m}$. The reported overall accuracy of the product calculated for a number of study sites over different environmental conditions is $88 \%$ [29].

- A series of 16-days MODIS composites at $250 \mathrm{~m}$ spatial resolution corresponding to the same dates of each of the AWiFS scenes. The original radiometrically calibrated and atmospherically corrected red wavelength reflectance MODIS are produced within the EFFIS pre-processing chain as $250 \mathrm{~m}$ rasters in LAEA/ETRS89 projection [30]. 
- CORINE Land Cover dataset (CLC) [31]. The CLC provides land use and land cover information at pan-European level. CLC is mostly produced on the basis of visual interpretation of high resolution remote sensing data including 44 land use/land cover classes mapped with a minimum mapping unit of 25 ha. The CLC 2006 update with a $100 \mathrm{~m}$ spatial resolution was used for this study. In the absence of definitive accuracy figures for CLC 2006, the figures for CLC 2000 provide a basic indication. The reliability of CLC 2000 at $95 \%$ confidence level was estimated at $87.0 \pm 0.7 \%[32]$.

In order to be in line with the spatial resolution of the IRS-AWiFS scenes all of the above datasets were resampled to $50 \mathrm{~m}$ rasters.

\section{Methodology}

The burned scar map for EU-MED from IRS-AWiFS scenes was created using a two stage classification approach [33]. This method was specifically designed for operational and automatic burned scar mapping over large regions with medium resolution imagery. The method proved to perform reliably on a variety of geographic conditions in Mediterranean Europe (Figures 2 and 3). It implements a two stage approach. Two stage burned scar mapping methods have been previously proposed in the literature $[2,34,35]$. They represent a more flexible alternative for analyses over large areas with very diverse conditions. In our case the first stage identifies burned scar seeds based on a non-parametric learning algorithm coupled with a bootstrap aggregation process. The second stage implements a region growing process to extend the area of the burned scars. This combination allows conservative seed estimation in the first stage that is subsequently balanced in the region growing process.

Figure 2. Flow chart of the two stage burned scar mapping approach.

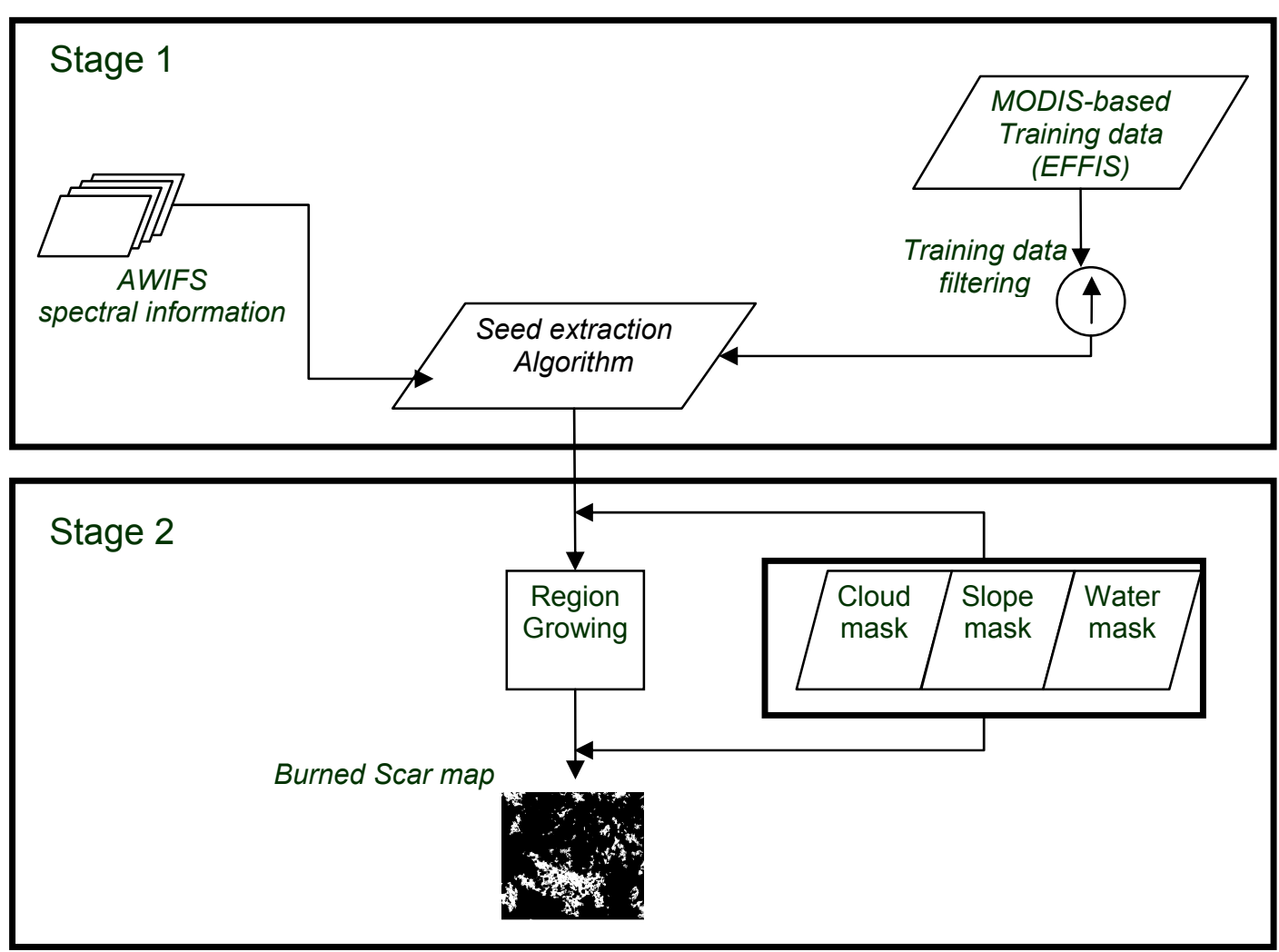


Figure 3. Burned scar mapping approach sequence for an area in northern Portugal ((ETRS89/LAEA projection). (A) AWIFS near infrared band; (B) Burned area seeds; (C) burned area after region growing. Unburned areas as represented in white color.

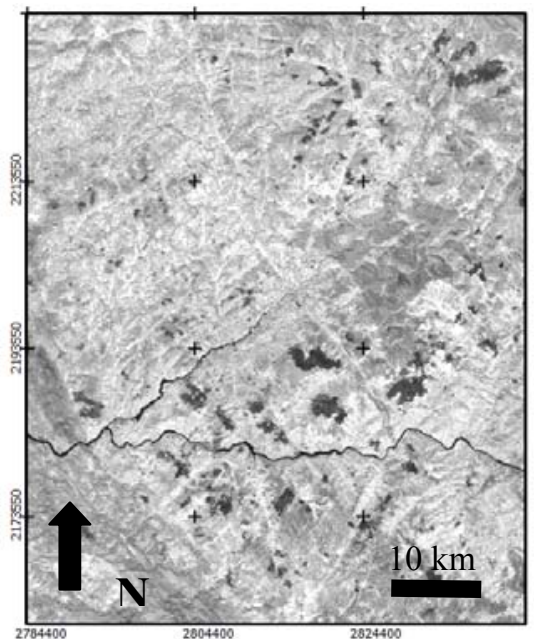

(A)

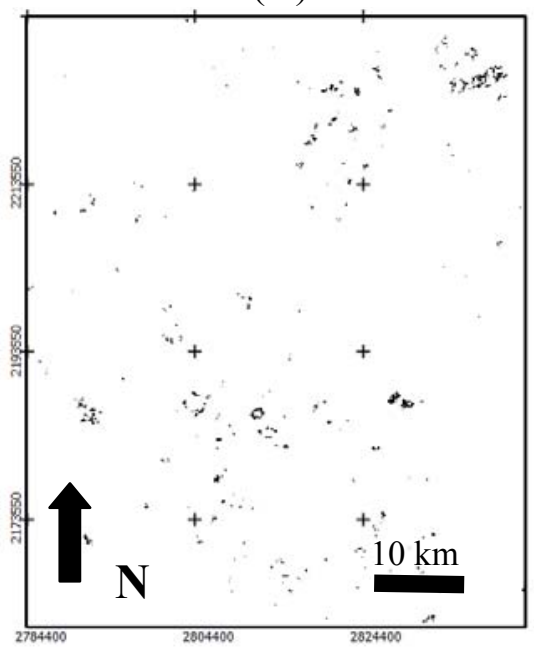

(B)

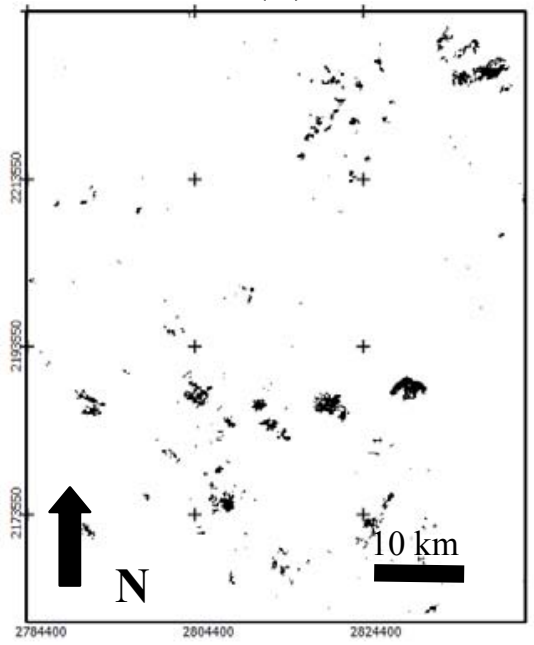

(C)

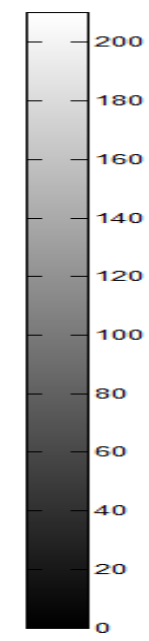

Unburned area

Burned area seeds
Unburned area

Burned area after region growing

Non-parametric classifiers make few assumptions about the data and are able to learn and model non-linear relationships. They do not need data fitting into a statistical distribution as linear classifiers 
and do not require a set initial parameters as threshold methods do. These characteristics make non-parametric algorithms a suitable alternative for burned area seed identification.

Previous recent studies have shown the improved performance of non-parametric algorithms such as support vector machines [36], regression trees or Artificial Neural Network (ANN) [33] for burned scar mapping. This study, building on previous in-house experience, uses an Artificial Neural Network as training algorithm. ANNs have the ability to learn patterns or relationships from given training data, generalize from such data [37] and provide a more powerful and adaptive non-linear mechanism [38]. These characteristics have fostered several studies exploring the application of ANN for the extraction of fire scar maps [39-41]. In order to minimize the potential instability of non-parametric classifiers due to the presence of outliers in the reference dataset, the ANN was coupled with a bootstrap aggregation routine [42]. Bootstrap aggregation averages predictions over a collection of bootstrap samples. These bootstrap samples are randomly selected subsets with replacement from the training data. The final pixel value is estimated as an aggregation of each bootstrap. By reducing variance and leaving bias unchanged, bootstrap aggregation (or bagging) can dramatically reduce the variance of unstable procedures and improve the overall prediction [43]. The second stage implements a region growing process to extend the burned scar seeds extracted in the first stage according to pre-defined similarity rules [44]. This allows for a detailed delineation of burned scars borders. As previously mentioned, the combination of region growing routines with other classification strategies substantially improves the result of the final classification, since the burned area patch identification routine can be designed to provide a conservative estimation of initial burned area seeds, reducing the misclassification errors [33].

The method was automatically applied to process each individual IRS-AWiFS scene sequentially. The burned EFFIS-RDA polygons were spectrally filtered to remove non burned pixels inside and near the borders of the burned polygons from the training dataset. Also, given the amplitude of the acquisition window for the IRS-AWiFS scenes, late summer fires might occur after the acquisition dates. For each IRS-AWiFS scene, forest fire events occurring after its acquisition date were not considered for the training of the burned scar algorithm. After filtering the EFFIS-RDA polygons, training data was extracted from pixels registered as burned and non-burned in the EFFIS-RDA polygons. The training pixels were randomly selected keeping the proportion of burned non-burned pixels within each IRS-AWiFS scene.

Features with low digital numbers in IRS-AWiFS bands such as shaded slopes, cloud shades or water are likely to be confused with burned scars [45]. In order to remove these features from the analysis, a series of masks was generated. A cloud mask for each IRS-AWIFS scene was created by comparing spectral digital numbers of land surfaces in the IRS-AWIFS scenes with its corresponding digital numbers in reference cloud free MODIS scenes acquired on the same dates of the IRS-AWIFS scenes [46]. Subsequently, a morphological operation together with a spectral threshold identified cloud shadows within the neighborhood of each cloud. A slope mask was defined to mask out shaded slopes that might have been misclassified as burned scars. The slope mask was created from a $100 \mathrm{~m}$ spatial resolution digital elevation model (DEM). Slopes higher than 10 degrees were defined as areas of potential shaded relief.

Digital numbers of water bodies in IRS-AWiFS wavelengths can be similar to those of burned scars. Therefore a water mask was used to remove water pixels from the burned scar classification. The FTYP 2006 was used for this purpose. The water class of FTYP2006 was applied to mask water 
bodies in the IRS-AWiFS scenes. The water class from the FTYP2006 allows a more detailed representation of water bodies, streams and coast lines than CLC water class, which is limited by the 25 ha minimum mapping unit of the CLC product.

Agricultural fires at the end of the summer are a common practice in some regions of Mediterranean Europe. These fires are also visible in the IRS-AWiFS scenes. Since the focus of this study is on forest fires, burned areas falling within arable land ("Non-irrigated arable land", "Permanently irrigated land", "Rice fields" CLC classes) were masked out from the analysis. These four masks were applied before and after the region growing stage. The first application prevented cloud shadow, shaded relief and water pixels misclassified as burned scar seeds by the learning algorithm to be classified as burned scars. The second application prevented burned scars started from burned scar seeds to grow into cloud shadow, shaded relief and water pixels. Although the applications of these masks implies that burned areas falling in cloud shadows, water and shaded slopes are not detected, the cost of underestimating burned areas on shaded slopes amply overcomes the potential overestimation of shaded slopes.

The final burned scar map is the result of compositing individual IRS-AWiFS maps. After masking out pixels with clouds, cloud shades, relief shades and water, the number of valid observations per pixel varies (Figure 1). Pixels were considered as burned in the final mosaic if they had been classified as such in at least one of the scenes. Vegetation senescence can result in near-infrared decreases similar to those caused by burning [17]. In areas dominated by dry senescent grass the region growing process of the burned scar detection algorithm results in a disproportionate growth of the original burned seeds. As a consequence, large extents can potentially be labeled as burned. This situation happens occasionally in Mediterranean islands largely covered by low vegetation. A final visual check was performed to remove these misclassifications.

\section{Results and Discussion}

The burned scar map from the IRS-AWiFS dataset displayed 901 burned scars larger than 10 ha with a total burned area of 145,100 ha. The map presents a higher frequency of small and medium size burned scars, gradually decreasing for larger burned scars (Figure 4). The spatial distribution of the burned scars is heterogeneous, with the highest densities recorded in North West of the Iberian Peninsula and clusters of burned scars in Sardinia and Greek islands.

The validation of burned scar products is challenging because of the difficulty of collecting independent, reliable and extensive reference data [47]. The use of paper maps from different sources [8], the visual interpretation or remote sensing-based products from higher resolution images $[47,48]$ have been proposed as viable alternatives in some cases. The absence of such a spatially consistent validation dataset for burned scars in Mediterranean Europe drove us to search for indirect alternatives to evaluate the performance of the IRS-AWiFS-based burned scar map. Although it is not a standard validation exercise, the inter-comparison of the IRS-AWiFS-based and the EFFIS-RDA burned scars maps can still provide some insights into the potential and limitations of both products. There are significant differences in the number of burned scars and the total burned area. For 2009, the EFFIS dataset listed circa 663 burned scars larger than 10 ha with a total burned area of 267,500 ha. The distribution of burned scars by area of both the IRS-AWiFS-based product and the EFFIS-RDA present a consistent spatial distribution and similar area frequencies (Figure 4). The rationale for a 
burned area estimate at a higher spatial resolution is based on the assumption that increased spatial detail would potentially result in an improved capability for the detection of smaller burned scars and a more precise perimeter of burned scars. The burned scar map produced from the IRS-AWiFS imagery acquired at the end of the 2009 European summer season shows that, in general, this burned scars map provides a more detailed delineation of burned area polygons. In addition, islands of unburned vegetation within the burned perimeters are better detected. This more detailed information has a considerable impact in annual burned area figures. Table 1 presents the comparison of the burned areas for the five largest forest fires in Mediterranean Europe during 2009.It shows that there are differences between the figures in the order of $20-50 \%$ for the medium and high spatial resolution methods. This is supported by a visual inspection of a number of medium and large size forest fires (Figure 5). Similar disparities have been previously reported by Zhang et al. [12] when comparing ETM+ and SPOT VEGETATION burned area estimates.

Figure 4. Burned area cumulative frequencies for MODIS-based EFFIS Rapid Damage Assessment (EFFIS-RDA) and IRS-AWiFS-based burned scar map.

\section{Burnt Area cumulative Frequencies}

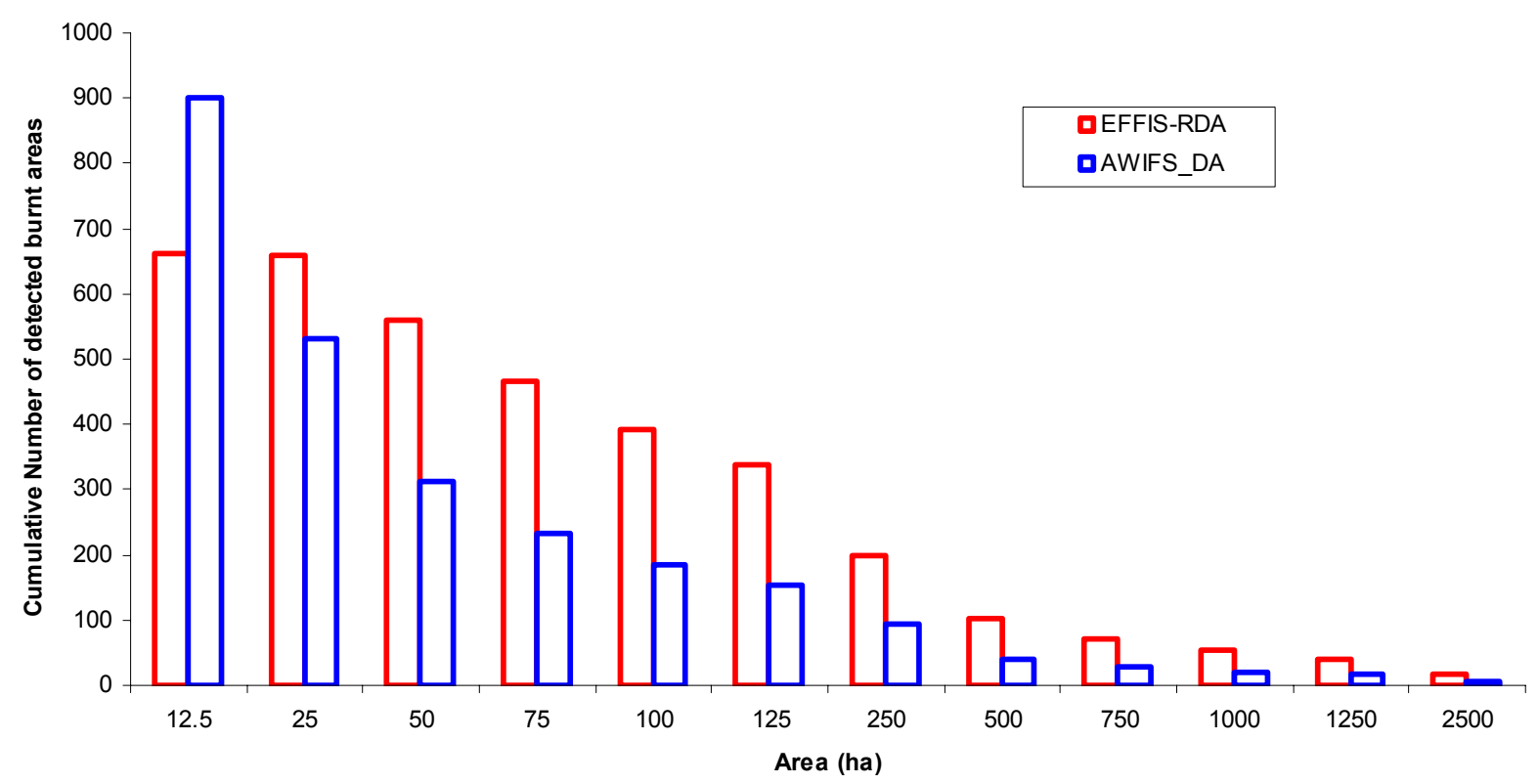

Table 1. Comparison of burned area estimates for the five largest forest fires in Mediterranean Europe in 2009 between MODIS-based European Forest Fire Information System Rapid Damage Assessment (EFFIS-RDA) and Advanced Wide Field Sensor (IRS-AWiFS)-based burned scar map.

\begin{tabular}{ccccc}
\hline ID & Country & RDA (ha) & DA (ha) & Area Difference (\%) \\
\hline 1 & Portugal & 12,075 & 7,262 & 39.86 \\
2 & Italy & 8,059 & 4,832 & 40.05 \\
3 & Spain & 7,570 & 4,446 & 41.27 \\
4 & Spain & 7,288 & 5,572 & 23.55 \\
5 & Spain & 7,165 & 3,525 & 50.80 \\
\hline
\end{tabular}


Figure 5. Difference in burned area estimation. Snapshots in northern Portugal (ETRS89/ LAEA projection) corresponding to (A) Near infrared IRS-AWiFS; (B) EFFIS-RDA burned scar; (C) IRS-AWiFS-based burned scars.

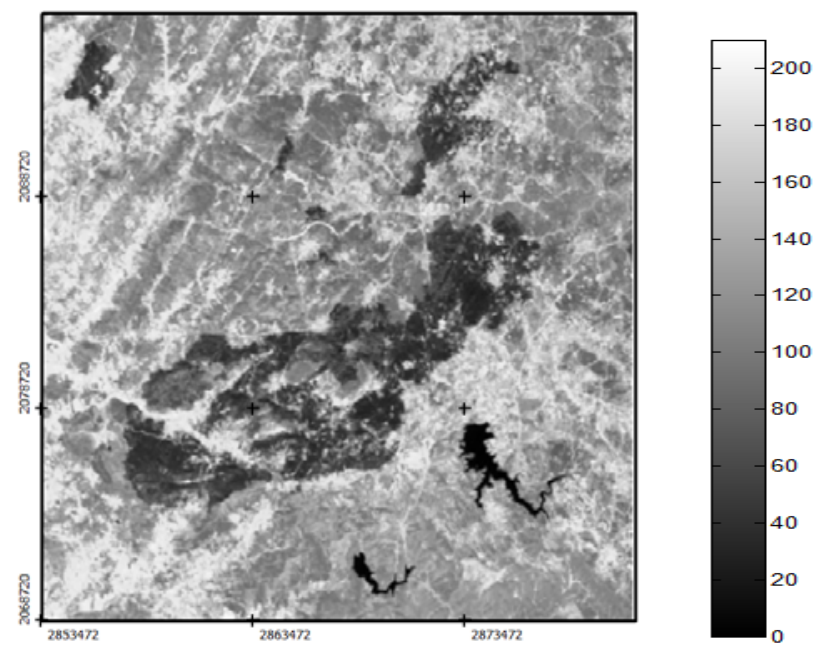

(A)

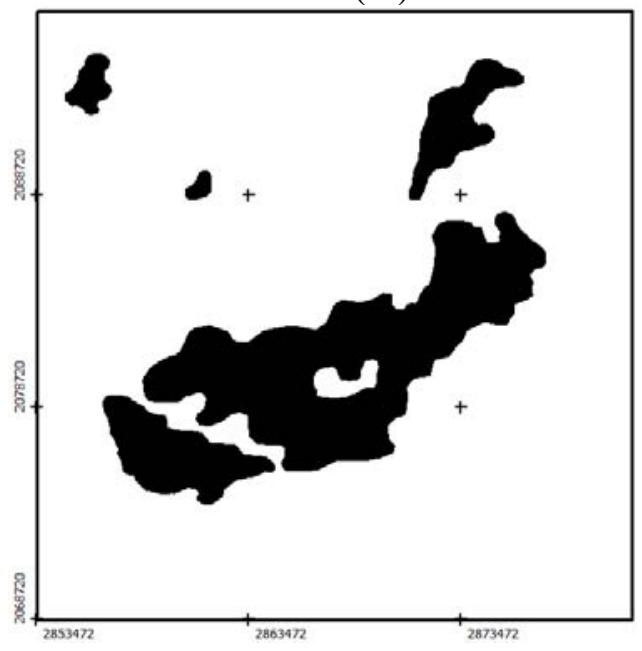

Unburned area

Burned area

(B)

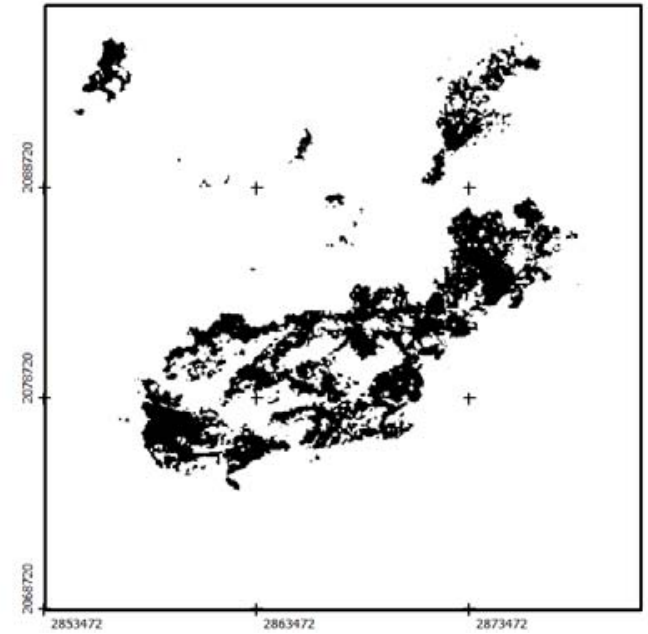

(C)

The results of this inter-comparison do, however, do not clearly support a consistent improved capability of the high spatial resolution imagery for detecting smaller burned scars. The detection rates 
of the IRS-AWiFS and the EFFIS-RDA maps show that nearly $75 \%$ of the burned scars smaller than 50 ha detected by IRS-AWiFS burned area map were recorded by EFFIS-RDA. This percentage gradually increases for medium and large burned scars: $80 \%$ of the burned areas larger than 100 ha and the totality of the burned areas larger than 500 ha detected by the IRS-AWiFS map detected by the EFFIS-RDA (Table 2). On the other hand, only $45 \%$ of the burned scars smaller than 50 ha detected in EFFIS-RDA are also detected by the IRS-AWiFS burned scar map. The figure gradually increases and more than $90 \%$ of burned scars larger than 2,000 ha mapped by EFFIS-RDA are also detected in the IRS-AWiFS map (Table 3), but some medium and large size forest fires detected in the EFFIS-RDA are still missed by the IRS-AWiFS map.

Table 2. Number of burned areas detected in IRS-AWiFS-based burned scar map and MODIS-based EFFIS Rapid Damage Assessment (EFFIS-RDA).

\begin{tabular}{cccc}
\hline $\begin{array}{c}\text { Burned Areas } \\
\text { Larger than (ha) }\end{array}$ & $\begin{array}{c}\mathbf{N}^{\mathbf{0}} \text { Detected Burned Areas } \\
\text { in EFFIS-RDA Map }\end{array}$ & $\begin{array}{c}\mathbf{N}^{\mathbf{0}} \text { Undetected Burned } \\
\text { Areas in IRS-AWiFS Map }\end{array}$ & $\begin{array}{c}\text { Percentage of Detection Agreement } \\
\text { (EFFIS-RDA as Reference) }\end{array}$ \\
\hline 10 & 663 & 376 & 43.29 \\
25 & 659 & 373 & 43.40 \\
50 & 561 & 308 & 45.10 \\
75 & 466 & 242 & 48.07 \\
100 & 391 & 193 & 50.64 \\
125 & 339 & 160 & 52.80 \\
250 & 200 & 76 & 62.00 \\
500 & 103 & 29 & 71.84 \\
750 & 71 & 17 & 76.06 \\
1,000 & 53 & 10 & 81.13 \\
1,250 & 39 & 6 & 84.62 \\
2,500 & 17 & 1 & 94.12 \\
\hline
\end{tabular}

Table 3. Number of burned areas detected in MODIS-based EFFIS Rapid Damage Assessment (EFFIS-RDA) and IRS-AWiFS-based burned scar map.

\begin{tabular}{cccc}
\hline $\begin{array}{c}\text { Burned Areas } \\
\text { Larger than (ha) }\end{array}$ & $\begin{array}{c}\mathbf{N}^{\mathbf{0}} \text { Detected Burned Areas } \\
\text { in IRS-AWiFS Map }\end{array}$ & $\begin{array}{c}\mathbf{N}^{\mathbf{0}} \text { Undetected Burned Areas } \\
\text { in EFFIS-RDA Map }\end{array}$ & $\begin{array}{c}\text { Percentage of Detection Agreement } \\
\text { (IRS-AWiFS as Reference) }\end{array}$ \\
\hline 10 & 901 & 439 & 51.28 \\
25 & 530 & 195 & 63.21 \\
50 & 312 & 79 & 74.68 \\
75 & 233 & 49 & 78.97 \\
100 & 186 & 30 & 83.87 \\
125 & 152 & 26 & 82.89 \\
250 & 93 & 10 & 89.25 \\
500 & 40 & 0 & 100.00 \\
750 & 27 & 0 & 100.00 \\
1,000 & 20 & 0 & 100.00 \\
1,250 & 17 & 0 & 100.00 \\
2,500 & 5 & 0 & 100.00 \\
\hline
\end{tabular}


In general, these figures show a good agreement for medium and large burned scars, although the higher resolution product misses some of these large scars (Figure 6). However, there are considerable discrepancies between both products for smaller burned scars (10-20 ha). While some of the small burned areas only detected in the IRS-AWiFS-based map are likely to correspond to the enhanced capacity for detecting small burned scars at higher spatial resolution. Higher spatial resolution generally implies, as a trade off, lower revisit periods and reduced spectral payloads. The reduced number of spectral bands limits the amount of available spectral information for automatic image classifications. This can explain some of the overestimations in the IRS-AWiFS burned scar map. Roy [17] showed that the highest spectral burned scar separability for MODIS bands over several ecosystems corresponds to near infrared (841-876 nm) and short wave infrared bands (1230-1250 nm). Since IRS-AWiFS lacks the equivalent short wave infrared band, most of the available information for burned scar detection is solely contained in the near infrared band. The concentration of the available information in a single band imposes a limitation. It has also been documented that vegetation senescence or vegetation removal can result in similar near infra red reflectance than burned scars [17].

Figure 6. Snapshots in North-west Spain (ETRS89/LAEA projection) corresponding to: (A) Near infrared IRS-AWiFS; (B) EFFIS-RDA burned scar; (C) IRS-AWiFS-based burned scars.
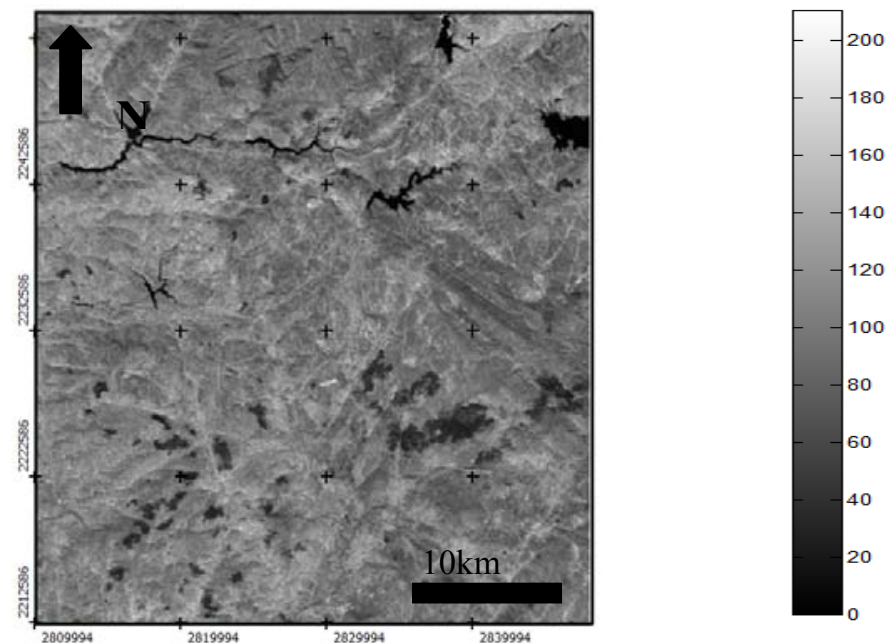

(A)

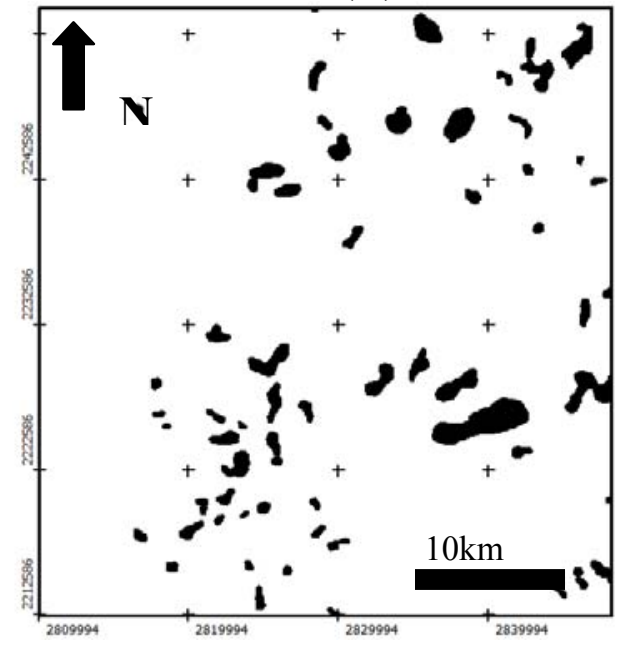

(B)
Unburned area

Burned area 
Figure 6. Cont.

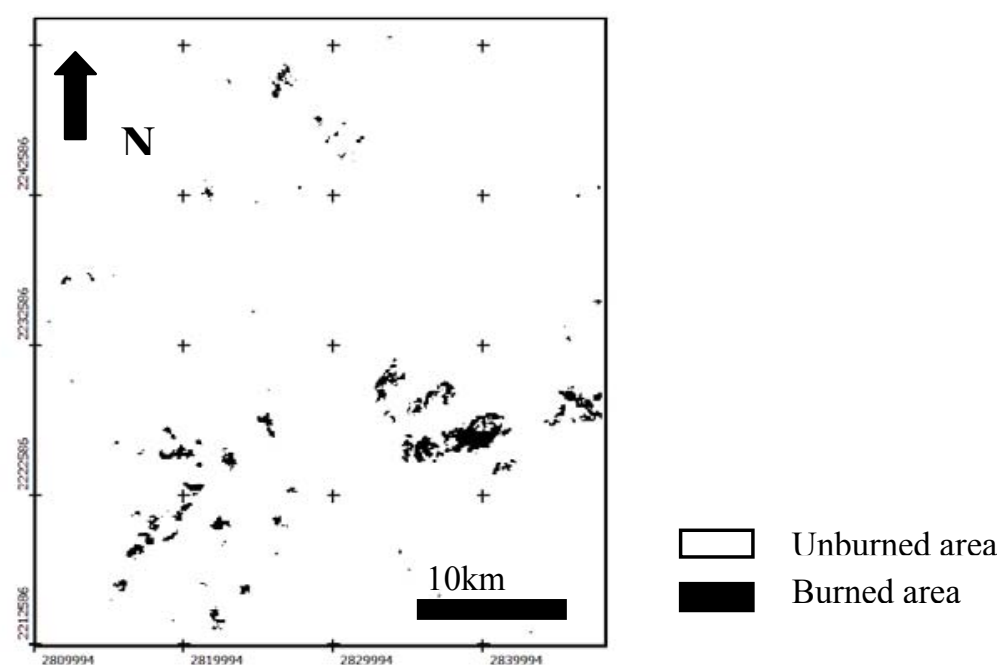

(C)

In addition, previous studies have also reported that shaded relief and shadows cast by cloud generally reduce digital numbers in all reflective bands [49] and consequently exhibit similar spectral profiles as those caused by fire [49]. The burned scar mapping method implemented in this study included a cloud, a cloud shadow and slope masks to minimize the commission error of burned scars. However, because of the spatial resolution of the AWiFS scenes, small clouds are likely to remain undetected. Besides, the spatial resolution of the available Digital Elevation Models does not allow masking out of all shaded slopes. As a consequence, cloud shade and shaded slope pixels can, under some conditions, be misclassified as burned seeds from which burned areas are generated. This can become a main source of burned area overestimations [33]. The overestimation of burned scars in the IRS-AWiFS product is responsible for the discrepancies in the detection of small burned scars in the EFFIS-RDA product.

There are also a number of explanations for the burned scars not detected in the IRS-AWiFS-based burned scar map. The higher spatial resolution also implies lower revisit period. As a consequence, a continuous image acquisition plan is not feasible. Instead, image acquisition must be concentrated at the end of the fire season. The lower revisit period of a higher resolution sensor is likely to result in incomplete coverage over large regions. In this study, the acquisition window for the IRS-AWiFS scenes spanned nine weeks (from mid August until late October). The 61 AWiFS scenes acquired during this period could not provide a full coverage of the study region and $11 \%$ of the study area was not covered by any AWiFS scene.

Furthermore, this acquisition window was not enough to obtain a cloud free coverage for the area for which imagery was available. Eight percent of the study region did not have any cloud free observation. Expanding the acquisition window into the high summer months would increase the chances of obtaining cloud free data, but at the expense of also increasing the number of late fires. Expanding the window into autumn months would increase the potential time lag between fire events and image acquisition, and the probability that the spectral signal of the burned scar fades away over time. This is particularly relevant for forest fires occurring in the first four months of the year. Early season fires (in areas of rapid vegetation recovery can be difficult to detect in late summer to autumn 
scenes. Although most of the forest fires occur on July and August, under drier weather conditions, early season fires are important in some regions of Mediterranean Europe.

In 2009, 21\% of the fires (accounting for an area of nearly 26,000 ha) recorded by EFFIS-RDA were labeled as early fires. Twenty five percent of them correspond to burned scars smaller than 250 ha. Almost all of them occurred in northwest Iberian Peninsula. The spectral response of the vegetation cover of burned scars in regions of high precipitation is likely to be spectrally similar to pre-fire conditions several months after the fire. Taking early season fires recorded in EFFIS-RDA as a reference, the analysis reveals that the detection rate for burned scars in the IRS-AWiFS-based map is, regardless of the size, $40 \%$ lower for early season fires. This represents a reduction in the detection rate (Table 3) with implications in both the number of detected burned scars and the total burned area estimated by the IRS-AWiFS-based product.

Late fires (forest fires occurring after the acquisition date of IRS-AWiFS scenes) also contribute to the underestimation of burned areas. They were present in 28 out of the 61 IRS-AWiFS scenes and around $8 \%$ of the burned area registered in EFFIS-RDA occurred after the acquisition date of the corresponding IRS-AWiFS scenes.

Some of the aforementioned limitations of burned scar mapping with medium resolution sensors could be overcome with a larger number of available scenes that would increase the chances of obtaining a full cloud free coverage of the area of interest. The possibilities for larger acquisitions will increase as the availability of medium resolution earth observation sensors grow [18]. In a scenario in which a large number of acquisitions are available for processing, the added value of burned scar mapping with medium resolution sensors must also include economic cost considerations. As an example, based on the cloud coverage calculated from the IRS-AWiFS dataset, three scenes covering the same area are required in order to guarantee less than $10 \%$ cloud coverage over the study area. Thus, since Mediterranean Europe is covered with circa 18 IRS-AWiFS scenes, a minimum of 54 scenes would be necessary. Still, a single coverage would not improve the under-detection of early season fires. Ideally we would need two sets of scenes: one acquired during the high summer months to identify early fires and another at the end of the summer to capture summer fires. Although the acquisition of the summer coverage would be restricted to regions in which a priori knowledge indicates a high frequency of early forest fires, a second dataset could double the number of scenes required. Therefore the acquisition cost associated to the increasing number of required scenes could still set a limitation to the application of medium resolution sensors for burned scar mapping.

\section{Conclusions}

A burned scar map for Mediterranean Europe based on IRS-AWiFS imagery acquired at the end of the 2009 fire season has been created with the objective of complementing and improving existing burned scar mapping products in EFFIS. This study is based on the assumption that higher spatial resolution of the satellite data results in an improved capability for the detection of small burned scars and a more precise delineation of burned areas. Today EFFIS-RDA is the only operational burned scar mapping initiative at pan-European level. Its semi automatic approach represents a time consuming and labor intensive effort during the fire season that provides a product of consistent performance. In 
contrast, the medium resolution burned scar map approach of this study represents a time efficient and automatic single-date analysis at the end of the fire campaign.

The burned scar map from the IRS-AWiFS dataset mapped 901 burned scars larger than 10 ha with a total burned area of 145,100 ha. The EFFIS dataset mapped circa 663 burned scars larger than 10 ha with a total burned area of 267,500 ha. In general, the inter-comparison of both products shows a good agreement for medium and large burned scars and an improvement in the delineation of burned scars. However, there are considerable discrepancies between both products for smaller burned scars (10-20 ha). These discrepancies are explained by several causes: Firstly, the absence of full imagery coverage and cloud free information; secondly the time lag between forest fire and image acquisition date and, thirdly, the presence of fires after the image acquisition dates can result in the under detection of a considerable number of burned scars of various sizes. On the other hand, the limited spectral resolution of the medium resolution sensors, the presence of undetected cloud shadows and shaded slopes is a potential source of misdetection of small burned scars.

Several of these limitations for burned scar mapping at medium spatial resolution are related to the present lack of sufficient medium resolution satellite imagery. These limitations are likely to be reduced in the near future as the availability of medium resolution earth observation sensors increase.

The combination of images from several similar sensors will give access to more complete and cloud free datasets at one or several periods during the fire season. This will improve the opportunities for burned scar mapping and monitoring at higher spatial resolution than existing systems. Until the moment this scenario becomes a reality, initiatives relying on coarse resolution satellite data remain a reasonable option for burned scar mapping and monitoring.

\section{Acknowledgements}

We would like to thank our colleagues Roberto Boca, Cristiano Giovando and Paolo Corti, in charge of the operational management of the European Forest Fire Information System Rapid Damage Assessment for their help providing the data use for this study. We would also like to thank two anonymous reviewers for their comments and constructive criticism that helped us to improve this manuscript.

\section{References}

1. Csiszar, I.; Justice, C.O.; Mcguire, A.D.; Cochrane, M.A.; Roy D.P.; Brown F. Land use and fires. In Land Change Science: Observing, Monitoring, and Understanding Trajectories of Change on the Earth's Surface; Gutman, G., Janetos, A.C., Justice, C.O., Moran, E.F., Mustard, J.F., Rindfuss, R.R., Skole, D., Turner, B.L., II, Cochrane, M.A., Eds.; Kluwer Academic Publishers: Dordrecht, The Netherlands, 2004.

2. Bastarrika, A. Cartografía Automática de Área Quemada a Nivel Local-Regional Mediante Algoritmos de Contexto Espacial (in Spanish). Ph.D. Thesis, University of Alcalá, Alcalá de Henares, Spain, 2009. 
3. Pereira, J.M.C.; Sá, A.C.L.; Sousa, A.M.O.; Silva, J.M.N.; Santos, T.N.; Carreiras, J.M.B. Spectral characterization and discrimination of burned areas. In Remote Sensing of Large Wildfires in the European Mediterranean Basin; Chuvieco, E., Eds.; Springer-Verlag: Berlin, Germany, 1999; pp. 123-138.

4. Chuvieco, E.; Congalton, R.G. Using cluster analysis to improve the selection of training statistics in classifying remotely sensed data. Photogramm. Eng. Remote Sensing 1998, 54, 1275-1281.

5. Jakubauskas, M.E.; Lulla, K.P.; Mausel, P.W. Assessment of vegetation change in a fire-altered forest landscape. Photogramm. Eng. Remote Sensing 1990, 56, 371-377.

6. Chuvieco, E.; Congalton, R.G. Mapping and inventory of forest fires from digital processing of TM data. Geocarto Int. 1988, 3, 41-53.

7. $\mathrm{Pu}, \mathrm{R}$; Gong, P. Determination of burned scars using logistic regression and neural network techniques from a single post-fire Landsat 7 ETM+ image. Photogramm. Eng. Remote Sensing 2004, 70, 841-850.

8. Kontoes, C.C.; Poilve, H.; Florsch, G.; Keramitsoglou, I.; Paralikidis, S. A comparative analysis of a fixed thresholding vs. a classification tree approach for operational burn scar detection and mapping. Int. J. Appl. Earth Obs. Geoinf. 2009, 11, 299-316.

9. Cochrane, M.A.; Souza, C.M. Linear mixture model classification of burned forests in the Eastern Amazon. Int. J. Remote Sens. 1998, 19, 3433-3440.

10. Polychronaki, A.; Gitas, I. Burned area mapping in Greece using SPOT-4 HRVIR images and object-based image analysis. Remote Sens. 2012, 4, 424-438.

11. Fraser, R.H.; Li, Z.; Chilar, J. Hotspot and NDVI Differencing Synergy (HANDS): A new technique for burned area mapping over boreal forest. Remote Sens. Environ. 2000, 74, 362-376.

12. Zhang, Y.-H.; Wooster, M.J.; Tutubalina, O.; Perry, G.L.W. Monthly burned area and forest fire carbon emission estimates for the Russian Federation from SPOT VGT. Remote Sens. Environ. 2003, $87,1-15$.

13. Tansey, K.; Grégoire, J.-M; Stroppiana, D.; Sousa, A.; Silva, J.M.N.; Pereira, J.M.C.; Boschetti, L.; Maggi, M.; Brivio, P.A.; Fraser, R.; Flasse, S.; Ershov, D.; Binaghi, E.; Graetz, D.; Peduzzi, P. Vegetation burning in the year 2000: Global burned area estimates from SPOT VEGETATION data. J. Geophys. Res.-Atmos. 2004, 109, D14S03.

14. Grégoire, J.-M.; Tansey, K.; Silva, J. M. N. The GBA2000 initiative: Developing a global burned area database from SPOT-VEGETATION imagery. Int. J. Remote Sens. 2003, 24, 1369-1376.

15. Simon, M.; Plummer, S.; Fierens, F.; Hoeltzemann, J.J.; Arino, O. Burned area detection at global scale using ATSR-2: The GLOBSCAR products and their qualification. J. Geophys. Res. 2004, 109, D14, D14 S02.

16. Plummer, S.; Arino, O.; Simon, M.; Steffen, W. Establishing a Earth observation product service for the terrestrial carbon community: The GlobCarbon initiative. Mitig. Adapt. Strategies Glob. Chang. 2006, 11, 97-111.

17. Roy, D.P.; Boschetti, L.; Justice, C.O.; Ju, J. The Collection 5 MODIS Burned Area Product: Global evaluation by comparison with the MODIS active fire product. Remote Sens. Environ. 2008, 112, 3690-3707. 
18. Sandau, R.; Paxton, L.; Esper, J. Trends and visions for small satellite missions. In Small Satellites for Earth Observation; Sandau, R., Röser, H.-P., Valenzuel, A., Eds.; Springer: Dordrecht, The Netherlands, 2008; pp. 27-39.

19. San-Miguel-Ayanz, J.; Camia, A. Forest fires at a glance: Facts, figures and trends in the EU. In Living with Wildfires: What Science can Tell Us? A Contribution to the Science-Policy Dialogue; Birot, Y., Ed.; EFI: Joensuu, Finland, 2009; pp. 11-18.

20. European Commission. Forest Fires in Europe 2008; EUR 23492 EN; Office for Official Publications of the European Communities: Luxembourg, 2009; p. 77.

21. Pereira, J.M.C.; Teresa, N.S. Fire Risk and Burned Area Mapping in Portugal; Direcção-Geral das Florestas DGF: Lisbon, Portugal, 2003.

22. Gonzalez-Alonso, F.; Merino-de-Miguel, S. Integration of AWiFS and MODIS active fire data for burn mapping at regional level using the Burned Area Synergic Algorithm (BASA). Int. J. Wildland Fire 2009, 18, 404-414.

23. FAO. FAO Global Ecological Zones; 2001. Available online: http://www.fao.org/geonetwork/srv/ en/main.home/ (accessed on 11 February 2011).

24. European Commission. Forest Fires in Europe 2009; EUR 24910 EN; Office for Official Publications of the European Communities: Luxembourg, 2010; p. 98.

25. IRS-P6 Data User's Handbook; IRS 6/NRSA/NDC/HB 10/03; National Remote Sensing Agency: Hyderabad, India, 2003.

26. INSPIRE. INSPIRE Directive; 2011. Available online: http://inspire.jrc.ec.europa.eu/ (accessed on 12 August 2011).

27. Farr, T.G.; Rosen, P.A.; Caro, E.; Crippen, R.; Duren, R.; Hensley, S.; Kobrick, M.; Paller, M.; Rodriguez, E.; Roth, L.; Seal, D.; Shaffer, S.; Shimada, J.; Umland, J. The Shuttle Radar Topography Mission. Rev. Geophys. 2007, 45, RG2004.

28. Rodriguez, E.; Morris, C.S.; Belz, J.E.; Chapin, E.C.; Martin, J.M.; Daffer, W.; Hensley, S. An Assessment of the SRTM Topographic Products; Technical Report JPL D-31639; Jet Propulsion Laboratory, Pasadena, CA, USA, 2005; pp. 143.

29. Kempeneers, P.; Sedano, F.; Seebach, L.; Strobl, P.; San Miguel, J. Data fusion of different spatial resolution remote sensing images applied to forest type mapping. IEEE Trans. Geosci. Remote Sens. 2011, 49, 4977-4986.

30. San-Miguel-Ayanz, J.; Pereira, J.M.C.; Boca, R.; Strobl, P.; Kucera, J.; Pekkarinen, A. Forest fires in the European Mediterranean Region: Mapping and analysis of burned areas. In Earth Observation of Wildland Fires in Mediterranean Ecosystems; Chuvieco, E., Ed.; Springer-Verlag: Berlin, Germany, 2009; pp. 189-204.

31. EEA. European Environmental Agency Data Service; 2010. Available online: http://www.eea.europa.eu/data-and-maps/data/ (accessed on 11 February 2011).

32. EEA. The Thematic Accuracy of Corine Land Cover, Assessment Using LUCAS (Land Use/Cover Area Frame Statistical Survey); Technical Report No 7/2006; 2006. Available online: http://www.eea.europa.eu/publications/technical_report_2006_7/ (accessed on 12 February 2012).

33. Sedano, F.; Kempeneers, P.; Strobl, P.; Vogt, P.; San Miguel, J. Towards a pan-European burnt scar mapping methodology based on single date high resolution optical remote sensing data. Int. J. Appl. Earth Obs. Geoinf. 2011, in press. 
34. Martin, P. Cartografía e inventario de incendios forestales en la Península Ibérica a partir de imágenes NOAA-AVHRR (in Spanish). Ph.D. Thesis, University of Alcalá, Alcalá de Henares, Spain, 1998.

35. Bastarrika, A.; Chuvieco, E.; Martín, M.P. Mapping burned areas from Landsat TM/ETM+ data with a two-phase algorithm: Balancing omission and commission errors. Remote Sens. Environ. 2011, 115, 1003-1012.

36. Petropoulos, G.P.; Kontoes, C.; Keramitsoglou, I. Burnt area delineation from a uni-temporal perspective based on Landsat TM imagery classification using Support Vector Machines. Int. J. Appl. Earth Obs. Geoinf. 2011, 13, 70-80.

37. Anderson J.A.; Rosenfeld, E. Neurocomputing: Foundations of Research; MIT Press: Cambridge, MA, USA, 1988.

38. Kimes, D.S.; Nelson, R.F.; Manry, M.T.; Fung, A.K. Attributes of neural networks for extracting continuous vegetation variables from optical and radar measurements. Int. J. Remote Sens. 1998, 19, 2639-2662.

39. Al-Rawi, K.R.; Casanova, J.L.; Calle, A. Burned area mapping system and fire detection system, based on neural networks and NOAA-AVHRR imagery. Int. J. Remote Sens. 2001, 22, 2015-2032.

40. Brivio, P.A.; Maggi, M.; Binaghi, E.; Gallo, I. Mapping burned surfaces in sub-Saharan Africa based on multi-temporal neural classification. Int. J. Remote Sens. 2003, 24, 4003-4018.

41. Sunar, F.; Özkan, C. Forest fire analysis with remote sensing data. Int. J. Remote Sens. 2001, 22, 2265-2277.

42. Breiman, L. Bagging predictors. In Machine Learning; Kluwer Academic Publishers: Hingham, MA, USA, 1996.

43. Hastie, T.; Thibsinari, R.; Friedman, J. The Elements of Statistical Learning. Data Mining, Inference and Prediction; Springer-Verlag: New York, NY, USA, 2001.

44. González, R.C.; Woods, R.E. Digital Image Processing, 2nd ed.; Prentice Hall: Upper Saddle River, NJ, USA, 2002.

45. Roy, D.P.; Lewis, P.E.; Justice, C.O. Burned area mapping using multi-temporal moderate spatial resolution data-A bi-directional reflectance model-based expectation approach. Remote Sens. Environ. 2002, 83, 263-286.

46. Sedano, F.; Kempeneers, P.; Strobl, P.; Vogt, P.; Seebach, L.; San Miguel, J. A cloud mask methodology for high resolution remote sensing data combining information from high and medium resolution optical sensors. ISPRS J. Photogramm. 2012, in press.

47. Roy, D.P.; Boschetti, L. Southern Africa Validation of the MODIS, L3JRC and GLOBCARBON Burned Area Products. IEEE Trans. Geosci. Remote Sens. 2009, 47, 1032-1044.

48. Holben, B.; Justice, C. An examination of spectral band ratioing to reduce the topographic effect in remotely sensed data. Int. J. Remote Sens. 1981, 2, 115-133.

(C) 2012 by the authors; licensee MDPI, Basel, Switzerland. This article is an open access article distributed under the terms and conditions of the Creative Commons Attribution license (http://creativecommons.org/licenses/by/3.0/). 